\title{
OFDM Channel Estimation Along with De- noising Approach under Small SNR Environment using SSA
}

\author{
E. Hari Krishna, K. Sivani and K. Ashoka Reddy
}

\begin{abstract}
In this paper, a de-noising approach in conjunction with channel estimation (CE) algorithm for OFDM systems using singular spectrum analysis (SSA) is presented. In the proposed algorithm, the initial $\mathrm{CE}$ is computed with the aid of traditional linear minimum mean square error (LMMSE) algorithm, and then further channel is evaluated by considering the low rank eigenvalue approximation of channel correlation matrix related to channel using SSA. Simulation results on bit error rate (BER) revealed that the method attains an improvement of $7 \mathrm{~dB}, 5 \mathrm{~dB}$ and $3 \mathrm{~dB}$ compared to common LSE, MMSE and SVD based methods respectively. With the help of statistical correlation coefficient $(C)$ and kurtosis $(k)$, the SSA method utilized to de-noise the received OFDM signal in addition to $C E$. In the process of denoising, the received OFDM signal will be decomposed into different empirical orthogonal functions (EOFs) based on the singular values. It was established that the correlation coefficients worked well in identifying useful EOFs only up to moderate $S N R \geq 12 d B$. For low $S N R<12 \mathrm{~dB}$, kurtosis was found to be a useful measure for identifying the useful EOFs. In addition to outperforming the existing methods, with this de-noising approach, the mean square error (MSE) of channel estimator is further improved approximately $1 \mathrm{~dB}$ more in SNR at the cost of computational complexity.
\end{abstract}

Index Terms-BER, Channel Estimation, OFDM, SVD, SSA, Wireless Communications.

\section{INTRODUCTION}

$\mathrm{O}$ rthogonal frequency division multiplexing (OFDM) has been accepted globally to cater the need of data rate requirement of multimedia communication systems. With advances in signal processing algorithms the multiple-input multiple-output (MIMO) with OFDM is considered as the vital

Manuscript received June 11, 2020; revised September 21, 2021. Date of publication January 31, 2022. Date of current version January 31, 2022. The associate editor prof. Joško Radić has been coordinating the review of this manuscript and approved it for publication.

E. Hari Krishna is with the Department of Electronics \& Communication Engineering, University College of Engineering, Kakatiya University, Kothagudem, Telangana, India (e-mail: hari etta@yahoo.co.in)

K. Sivani is with the Department of Electronics \& Instrumentation Engineering, Kakatiya Institute of Technology \& Science, Warangal, Telangana, India (e-mail: sivanik234@gmail.com).

K. Ashoka Reddy is with the Department of Electronics \& Communication Engineering, Kakatiya Institute of Technology \& Science, Warangal, Telangana, India (e-mail: kareddy.iitm@gmail.com).

Digital Object Identifier (DOI): 10.24138/jcomss.v18i1.1082 methods [1]-[3]. Channel estimation (CE) plays a key role in these systems. In [1], the channel correlation matrix is made low rank by singular value decomposition (SVD) was presented to reduce the complexity of channel matrix inversion. Interpolation methods with pilot-based methodologies i.e. like block-type and comb-type arrangements are widely used for OFDM CE to better identify the channel properties [2]-[5]. These methods result in reduced efficiency because of transmission of training data additionally to the original data. Mathematical signal processing operations such as least squares (LS) and minimum mean square error (MMSE) estimators were deployed for OFDM CE [7]-[8]; majority of $\mathrm{CE}$ methods are the modified forms of LS estimation (LSE) and MMSE. To reduce the computational complexity, both time and frequency interpolation is employed for massive MIMO-OFDM systems based on DFT with utilizing different pilot patterns is presented [9]. The Krylov subspace technique with multi-stage nested Wiener filter (MSNWF) for low rank approximation is presented in [10] for OFDM CE. All forms of OFDM CE methods and its challenges were best illustrated in [11]-[12]. A different strategy of pilot patterns along with basic OFDM blocks was presented in [13] for OFDM CE under multipath fading channels, it jointly estimates the channel and carrier frequency offset (CFO) in a semi blind approach.

Without use of channel statistics and pilot tones, a coded neural network based method was presented in [14]. To further improve the accuracy of OFDM CE, lower modulation schemes with blind CE [15], and low rank approximation for OFDM CE [16] were presented. In similar lines, a novel blind subspace approach was presented in [17] by combining minor component analysis (MCA) and independent component analysis (ICA). LMMSE estimation using artificial channel attempts made in [18], which eliminates the channel covariance matrix. De-noising approaches based OFDM CE was presented in [19]-[20], which inspired us to use singular spectrum analysis (SSA) technique in conjunction with statistical analysis for $\mathrm{CE}$ and de-noising. In order to reduce the complexity of algorithms, learning automata based channel estimation was presented in [21]. MIMO-OFDM channel estimation using compressive sensing based LSE and MMSE was described in [22] and the nonlinear mode decomposition 
method and its application to wireless communication was presented in [23].

In [24] the de-noising of received OFDM symbol with decomposed highly correlated empirical orthogonal functions (EOFs) using statistical correlation analysis, worked well for high signal to noise ratios (SNR). But under small SNR, the performance is degraded. In order to improve the performance even under small SNR scenario, we proposed the use of statistical kurtosis analysis in addition to correlation, which was presented in the next sections. Section II describes the mathematical background of basic wireless OFDM transceiver system, section III presents the proposed signal processing method based on SSA and its use in channel estimation (CE) along with de-noising. Finally, section III depicts simulation results and conclusions in section IV.

\section{WIRELESS OFDM TRANSCEIVER SYSTEM}

The typical wireless OFDM transmitter, as depicted in fig.1, modifies the input binary data $b_{T}$ onto PSK/QAM mapper $F_{b}(k)$ and then implements IFFT on parallel transformed data.

$$
f_{b}(n)=\sum_{k=0}^{N-1} F_{b}(k) e^{j 2 \pi n k / N}, n=0,1,2, \ldots . . N-1
$$

where $N$ is the number of sub carriers. For data $f_{b}(n)$, the cyclic prefix (CP) is added to ensure zero ISI, $f_{c p}(n)$ is then changed to a domain suitable for wireless OFDM broadcasting.

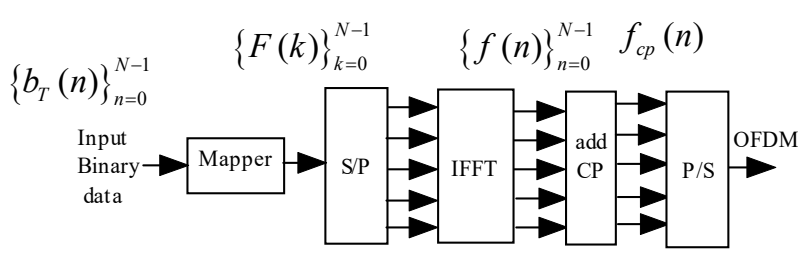

Fig. 1. Typical OFDM transmitter model

Figure 2 shows the receiver model showing proposed channel estimation and de-noising blocks using SSA. The digital converted signal at OFDM receiver can be viewed as

$$
y_{R}(n)=f_{c p}(n) \otimes h_{C I R}(n, k)+w(n)
$$

where $\otimes$ symbol indicates the convolution; $w(n)$ and $h_{C I R}(n)$ are additive white Gaussian noise (AWGN) with zero mean and unit variance and the channel impulse response (CIR) to be estimated respectively. In general, the channel is non-linear time varying wireless multipath fading channel and the $h_{C I R}(n)$ can be modeled as

$$
h(t, \tau)=\sum_{M=0}^{N-1} \tilde{a}_{M}(t) e^{j \omega_{M}(t)} \delta\left(t-\tau_{M}(t)\right)
$$

where, $k_{i}, \alpha_{i}(n)$ and $w(n)$ are delay incurred, corresponding attenuation and unit sample response occurred in $i^{\text {th }}$ path. $\omega_{M}(t)$ represents the angular Doppler frequency shift due to time varying. Finally, the received OFDM signal $y_{R}(n)$ is represented in frequency domain as i.e. Fourier transform of eq (2)

$$
Y_{R}(k)=F_{c p}(k) H_{C I R}(k)+W(k)
$$

The $Y_{R}(k)$ is now used for channel estimation. The time varying CIR is to be properly estimated to perfectly recover the original transmitted binary sequence.

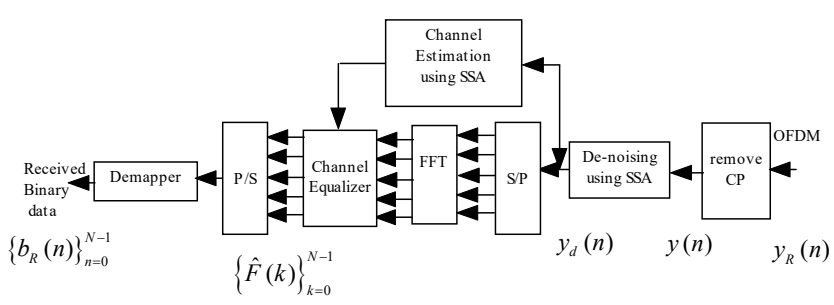

Fig. 2. OFDM Receiver model showing propsed channel estimation and denoising blocks using SSA

Broom-head and King [25]-[26] developed the methodological steps for SSA as shown in Fig. 3:

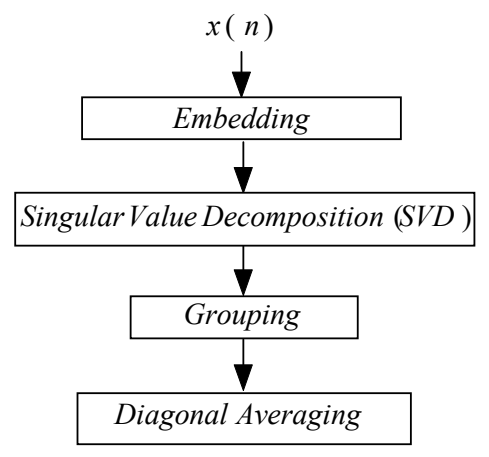

Fig. 3. SSA processing steps

As depicted in Fig. 3, the embedding and SVD are part of decomposition; grouping and diagonal averaging comes under reconstruction stage.

\section{A. Decomposition}

\section{i. $\quad$ Embedding}

The first step in the decomposition step of basic SSA algorithm is the embedding, where the data is changed into the trajectory matrix consists of overlapped data segments. Let the data be $x=\left\{x_{1} \ldots x_{n}\right\}$. In the embedding step, window length $L$ is considered in such a way that, where $2<L<N / 2$ to embed the initial data. $T_{X}$ is written as

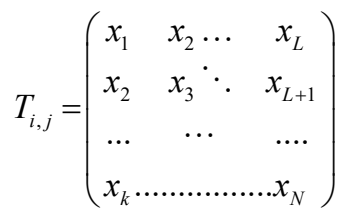

\section{ii. Singular value decomposition (SVD)}

After the embedding step, apply SVD to the trajectory matrix $T_{X}$ and obtain the decomposed trajectory matrices $T_{i}$ for 
$i=1, \ldots L$. The outcome of applying SVD to $T_{X}$ decomposes as $T_{X}=U V D^{\prime}$ called as eigentriples. $U_{i}$ for $1<i<L$ is a $K \times L$ orthogonal matrix. $D_{i}$ for $1<i<L$ is a diagonal matrix order of $L$. $V_{i}$ for $1<i<L$ is $L \times L$ square orthonormal matrix. In this step $T_{X}$, has $L$ many singular values which are $\sqrt{\lambda_{1}}>\sqrt{\lambda_{2}} \ldots \ldots \ldots \sqrt{\lambda_{L}}>$ thus the $i^{\text {th }}$ eigentriple of $T_{i}$ can be written as $U_{i} \times \sqrt{\lambda_{i}} \times V_{i}^{T}$ for $i=1,2, \ldots, d$, So the trajectory matrix $T_{X}$ can be denoted as

$$
\begin{aligned}
T_{x} & =T_{1}+T_{2}+\ldots . .+T_{d} \\
& =U_{1} \sqrt{\lambda_{1}} V_{1}^{T}+\ldots .+U_{d} \sqrt{\lambda_{d}} V_{d}^{T} \\
& =\sum_{i=1}^{d} U_{i} \sqrt{\lambda_{i}} V_{i}^{T}
\end{aligned}
$$

\section{B. Reconstruction}

\section{i. Regrouping}

The grouping step of the reconstruction stage is decomposing the $L \times K$ matrix $T_{i}$ into subgroups. The grouping step of the reconstruction stage is a partition of the set of indices $\{1,2, \ldots . d\}$ into the collection of $\mathrm{m}$ disjoint subsets of $I=\left\{I_{1}, \ldots \ldots \ldots I_{m}\right\} . T_{I_{i}}$ is a sum of $T_{j}$, where $\mathrm{j} \in I_{i}$.so $T_{x}$ can be expanded as

$$
\begin{aligned}
T_{x} & =\overbrace{T_{1}+\ldots \ldots . .+T_{L}}^{S V D} \\
& =\overbrace{T_{I_{i}}+\ldots \ldots . . T_{I_{m}}}^{\text {Grouping }}
\end{aligned}
$$

\section{ii. Diagonal Averaging}

In this step is to transform the grouping matrices $T_{I_{i}}$ into a new time series of length $N$.

$$
T_{i, j}= \begin{cases}\frac{1}{s-1} \sum_{j=1}^{s-1} x_{j, s-j} & \text { for } 2 \leq s \leq L \\ \frac{1}{L} \sum_{j=1}^{L} x_{j, s-j} & \text { for } L+1 \leq s \leq K+1 \\ \frac{1}{N-s+2} \sum_{j=s-K}^{N-s+2} x_{j, s-j} & \text { for } K+2 \leq s \leq N+1\end{cases}
$$

\section{Proposed Signal Processing Method BASEd on SSA}

In this proposed method, similar lines as done in SVD, using the traditional LMMSE method the initial CE will be done; and then the channel will be tracked by performing the rank reduction of channel correlation matrix for optimum complexity using SSA. Previously in [1], the approximation is done using SVD, but it was assumed that the channel was slow fading i.e. the channel is unaltered during broadcast of one OFDM signal. Here, that assumption is eliminated in this proposed method.

\section{A. LMMSE Estimation}

Let $b_{T}(n)=$ the transmitted symbol, $y_{R}(n)=$ the received symbols, $h_{C I R}=$ the assumed CIR, $\hat{h}_{C I R}=$ the estimated CIR using proposed SSA based method based and $\hat{h}_{\text {CIRL }}=$ the estimated CIR using the least squares (LS) estimated channel,

$$
\begin{aligned}
& Y=\left[y_{R}(0), y_{R}(1), \ldots \ldots \ldots \ldots y_{R}(N-1)\right]^{T} \\
& B=\left[b_{T}(0), b_{T}(1), \ldots \ldots \ldots \ldots b_{T}(N-1)\right]^{T} \\
& h_{C I R}=\left[h_{C I R}(0), h_{C I R}(1), \ldots \ldots \ldots \ldots h_{C I R}(N-1)\right]^{T} \\
& \hat{h}_{C I R}=\left[\hat{h}_{C I R}(0), \hat{h}_{C I R}(1), \ldots \ldots \ldots \ldots \hat{h}_{C I R}(N-1)\right]^{T} \\
& \hat{h}_{C I R L}=\left[\hat{h}_{C I R L}(0), \hat{h}_{C I R L}(1), \ldots \ldots \ldots \ldots \hat{h}_{C I R L}(N-1)\right]^{T}
\end{aligned}
$$

The LMMSE estimated CIR is [1]

$$
\hat{H}_{C I R L E}=R_{H_{C I R} \hat{H}_{C I R L}} R_{\hat{H}_{C I R L}^{-1} \hat{H}_{C I R L}} \hat{H}_{C I R L}
$$

The vector notation of above defined variables is given below

$$
=R_{H_{C I R} H_{C I R}}\left(R_{H_{C I R} H_{C I R}}+\sigma_{n}^{2}\left(B B^{H}\right)^{-1}\right)^{-1} \hat{H}_{C I R L}
$$

$\hat{H}_{C I R L}$ is transform domain LS estimated CIR

$$
\begin{aligned}
& R_{H_{C I R} H_{C I R}}=E\left\{H_{C I R} H_{C I R}^{H}\right\} \\
& R_{H_{C I R} \hat{H}_{C I R L}}=E\left\{H_{C I R} \hat{H}_{C I R L}^{H}\right\} \\
& R_{\hat{H}_{\text {CIRL }} \hat{H}_{\text {CIRL }}}=E\left\{\hat{H}_{\text {CIRL }} \hat{H}_{\text {CIRL }}^{H}\right\}
\end{aligned}
$$

To reduce the complexity, the matrix inversion in equation (10), is replaced with average of communicated data [1]. Initially, CE is computed with the aid of traditional LMMSE, and then further channel is evaluated using matrix related to channel

$$
\hat{H}_{C I R}=R_{H_{C I R} H_{C I R}}\left(R_{H_{C I R} H_{C I R}}+\frac{\beta}{S N R} I\right)^{-1} \hat{H}_{C I R L}
$$

Finally, the estimated CIR is uniquely proportional to correlation matrix, so the same $R_{H_{C I R} H_{C I R}}$ is now reduced to low rank using SVD method [1]. In the SVD method, it was assumed as that the channel was slow fading i.e. the channel is unaltered during broadcast of one OFDM signal. So, here a low rank approximation is used with SSA. SSA can be viewed as SVD with overlapped data segment, which eliminates the above mentioned assumption.

\section{B. Rank reduction of correlation matrix with the aid of SSA}

As per the methodological steps of SSA, the matrix $R_{H_{C I R} H_{C I R}}$ is modified with its overlapping segments. Then SVD of channel correlation matrix is given by

$$
R_{H_{C R} H_{C I R}}=U \Lambda U^{H}
$$

$U=\left[u_{0}, u_{1}, \ldots \ldots \ldots \ldots u_{N-1}\right], u_{0}, u_{1}, \ldots \ldots u_{N-1}$ are column vectors. $\Lambda$ contains all decomposed singular values in decreasing order as diagonal matrix $\Lambda=I\left[\lambda_{0}, \lambda_{1}, \ldots \ldots \ldots \ldots \lambda_{N-1}\right]$. As the first few 
represent the important components and last few related to unimportant one, so now the rank of matrix is reduced by considering first $p$ singular values and remaining made to forcible zeros. Then equation (15) gets simplified to

$$
\hat{H}_{C I R}=U \Gamma U^{H} \hat{H}_{C I R L}
$$

where $\Gamma$ contains modified diagonal matrix containing first few $p$ values and remaining are zeros. This (16) represents the estimated channel.

\section{SSA based denoising of received OFDM symbol using Pearson correlation and kurtosis}

Singular spectrum analysis (SSA) decomposes the received OFDM symbol into different empirical orthogonal function (EOF) based on eigenvalue driven singular value decomposition (SVD). First few EOFs represent the important components of the signal and last EOFs represent the unimportant or noisy components of the signal. The selection of EOFs is based on either correlation or kurtosis values.

Under high SNR scenarios, EOFs with high correlation values represents the required signal and low correlation value indicates undesired noisy versions. In order to de-noise the OFDM signal, a correlation threshold is proposed here for selection of signal EOFs.

For effective de-noising, at typically low SNR situations, in addition to correlation coefficient we propose to use another statistical measure kurtosis is also used for selection of appropriate EOFs. In general, a high value of kurtosis indicates random component present in EOF. So, eliminating EOFs having high kurtosis values amounts reducing the random noise component present in the received OFDM symbol. For this purpose, a kurtosis threshold on is proposed for selection of useful number of EOFs.

The subsequent steps are proposed to de-noise the OFDM signal. The received signal is disintegrated into empirical orthogonal functions (EOF). EOFs will be generated by considering different eigenvalues during reconstruction process. By considering the best EOFs, the modified OFDM symbol will be constructed, which represents de-noised version. The best available EOF is selected based on the Pearson correlation $\left(C_{X Y}\right)$, a statistical measure.

$$
C_{X Y}=\frac{\operatorname{cov}(X, Y)}{\sigma_{X} \sigma_{Y}}=\frac{\sum_{i=1}^{n}\left(x_{i}-\bar{x}\right)\left(y_{i}-\bar{y}\right)}{\sqrt{\sum_{i=1}^{n}\left(x_{i}-\bar{x}\right)^{2}} \sqrt{\sum_{i=1}^{n}\left(y_{i}-\bar{y}\right)^{2}}}
$$

where $\bar{x}, \bar{y}$ represent the mean of $x$ and $y$ respectively.

The correlation will be computed for all EOFs with recieved signal.

\section{(i) Threshold for selection of reliable EOFs}

The threshold for selection of signal EOFs is presented here using mean and stanadard deviation (std) of all evaluated correlation values.

$$
\begin{aligned}
\sigma_{\text {th_c }}= & \operatorname{mean}\left(C_{1}, C_{2}, \ldots, C_{i} \ldots C_{N-1}\right) \\
& -\operatorname{std}\left(C_{1}, C_{2}, \ldots, C_{i} \ldots C_{N-1}\right)
\end{aligned}
$$

where, $C_{i}$ is the correlation value of $i^{\text {th }}$ EOF with received signal; $i=1,2 \ldots N-1$. So, as part of the de-noising process the EOFs above the computed threshold will be considered.

\section{(ii) Kurtosis for selection of appropriate EOFs}

For effective denoising, at typically low SNR situations, in addition to correlation coefficient we propose to use another statistical measure kurtosis $(k)$ is also used for selection of appropriate EOFs.

$$
k=\frac{E(x-\mu)^{4}}{\sigma^{4}}-3
$$

where $\mu, \sigma$ are the mean and standard deviation of $x$. In general, the randomness of a typical signal can be measured by means Kurtosis.

\section{(iii) Threshold on Kurtosis for selection of reliable EOFs}

In general a high value of kurtosis indicates random component present in EOF. So, eliminating EOFs having high kurtosis values amounts reducing the random noise component present in the received OFDM symbol. For this purpose, a threshold $\left(\sigma_{t h_{-} k}\right)$ on kurtosis is proposed for selection of useful number of EOFs. This threshold $\left(\sigma_{t h-k}\right)$ is taken as mean of kurtosis values plus the stanadard deviation of kurtosis values.

$$
\begin{aligned}
\sigma_{t h \_k}= & \text { mean }\left(k_{1}, k_{2}, \ldots . . k_{i}, \ldots . k_{N-1}\right) \\
& +\operatorname{std}\left(k_{1}, k_{2}, \ldots . . k_{i}, \ldots . k_{N-1}\right)
\end{aligned}
$$

where $k_{i}$ is kurtosis value of $i^{t h}$ EOF component; $i=1,2 \ldots . N-1$.

The de-noised signal $\left(y_{d}(n)\right)$ is reconstructed using selected EOFs based on the $\sigma_{t h_{-} c}, \sigma_{t h_{-} k}$ values. This $y_{d}(n)$ serves as input to the channel estimation algorithm proposed in this paper. The performance of the proposed channel estimation method is studied through different simulations presented in the following section.

\section{SimUlation ResUlts AND ANALYSIS}

The simulation results and its analysis were presented in this section. To evaluate the performance of the presented SSA based technique, the subsequent simulation parameters, are utilized. In simulations, different types of noisy scenarios were considered, such as impulsive noise and Gaussian noise. During de-noising, the received signal is decomposed in to their equivalent EOF components. Correlation co-efficient $(C)$ and kurtosis $(k)$ values for all EOFs are calculated and tabulated in Table II at $S N R=23 d B$.

Correlation co-efficient between each decomposed EOF and received signal $k u$ of each decomposed EOF For the correlation values shown in Table II, mean is 0.49 , standard deviation is 0.2144 and computed threshold as per (18) is $\sigma_{\text {th_c }}=0.2756$. 
TABLE I. Simulation Parameters Utilized For Presented SSA BASED TECHNIQUE VALIDATION [11]

\begin{tabular}{cc}
\hline Simulation Parameters & Considered values \\
\hline Carrier Frequency & $5 \mathrm{GHz}$ \\
System bandwidth & $80 \mathrm{MHz}$ \\
Sub carrier spacing & $6.83 \mathrm{kHz}$ \\
Modulation & BPSK, QPSK, $16 \mathrm{QAM}$ and $64 \mathrm{QAM}$ \\
N-point IFFT & 256 - point IFFT \\
Length of Cyclic Prefix & 16 samples \\
Doppler Frequency & $100 \mathrm{~Hz}, 500 \mathrm{~Hz}$ and $1000 \mathrm{~Hz}$ \\
\hline
\end{tabular}

TABLE II. CALCULATED CORRELATION AND KURTOSIS VALUES OF DECOMPOSED EOFS @ SNR=23DB

\begin{tabular}{llllllll}
\hline \hline & $\mathbf{E}_{\mathbf{1}}(\mathbf{n})$ & $\mathbf{E}_{2}(\mathbf{n})$ & $\mathbf{E}_{3}(\mathbf{n})$ & $\mathbf{E}_{4}(\mathbf{n})$ & $\mathbf{E}_{5}(\mathbf{n})$ & $\mathbf{E}_{\mathbf{6}}(\mathbf{n})$ & $\mathbf{E}_{7}(\mathbf{n})$ \\
\hline \hline $\boldsymbol{C}$ & 0.721 & 0.7053 & 0.5621 & 0.5383 & 0.4945 & 0.2518 & 0.1562 \\
$\boldsymbol{k}$ & 2.0271 & 3.5214 & 5.1617 & 4.7306 & 5.3852 & 7.1716 & 7.2764 \\
\hline
\end{tabular}

Hence, the EOFs having correlation values above $\sigma_{t h \_}=0.2756$, i.e., the EOFs $E_{l}(n)$ to $E_{5}(n)$ are selected for de-noising. Similarly, in case of kurtosis values, mean is 5.0391, standrad deviation is 1.8779 and computed threshold as per (20) is $\sigma_{t h_{-} k}=6.917$. Hence, the EOFs having kurtosis values below $\sigma_{\text {th_k }}=6.917$, i.e., the EOFs $E_{l}(n)$ to $E_{5}(n)$ are selected for de-noising. It can be noticed here that both thresolds $\sigma_{t h_{-} c}$ and $\sigma_{t h_{-} k}$ selected same five EOFs $\left(E_{l}(n)\right.$ to $\left.E_{5}(n)\right)$ for eleimination of uncorrelated and random noise components from the received OFDM symbol.

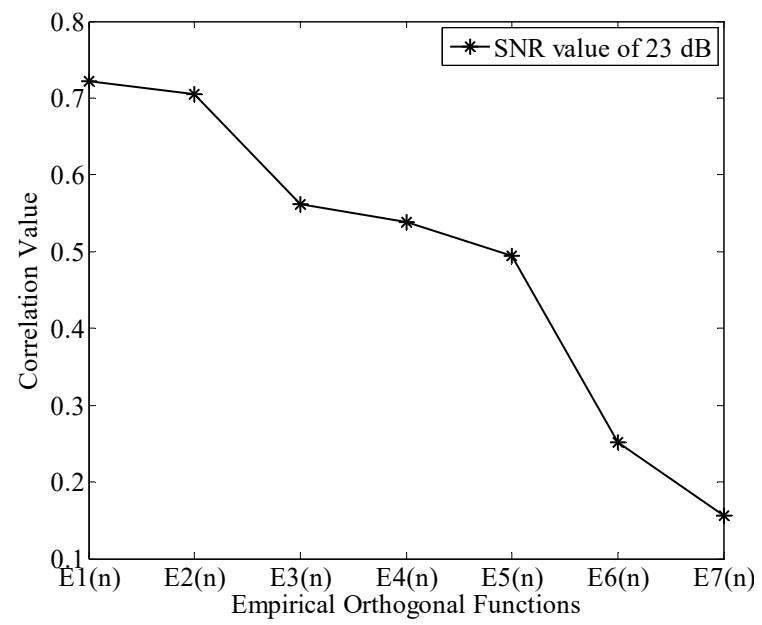

Fig. 4. Correlation values of different EOF with received OFDM symbol

At high SNRs, the correlation values, presented in Table-II, are monotonically decreasing in nature, as shown in Fig. 4. This makes the selection of EOFs very easy.

At low values of SNR say $3 \mathrm{~dB}$, for same OFDMsymbol, the calculated correlation and kurtosis values are presented in Table-III. The correlation values, as shown in Fig. 5, are not monotonically decreasing. This makes the selection of EOFs difficult. Here the mean of correlation values is 0.4678 , standard deviation is 0.2671 and calculated threshold $\sigma_{\text {th_c }}=0.2007$.

TABLE III. CALCULATED CORRELATION AND KURTOSIS VALUES AT 3DB

\begin{tabular}{llllllll}
\hline \hline & $\mathbf{E}_{\mathbf{1}}(\mathbf{n})$ & $\mathbf{E}_{\mathbf{2}}(\mathbf{n})$ & $\mathbf{E}_{\mathbf{3}}(\mathbf{n})$ & $\mathbf{E}_{\mathbf{4}}(\mathbf{n})$ & $\mathbf{E}_{\mathbf{5}}(\mathbf{n})$ & $\mathbf{E}_{\mathbf{6}}(\mathbf{n})$ & $\mathbf{E}_{\mathbf{7}}(\mathbf{n})$ \\
\hline \hline $\mathbf{P C C}$ & 0.1961 & 0.8192 & 0.6591 & 0.6294 & 0.5892 & 0.1944 & 0.1873 \\
$\mathbf{k u}$ & 2.1193 & 3.4742 & 5.1738 & 4.7576 & 5.4735 & 7.564 & 7.8764 \\
\hline
\end{tabular}

As per the explained procedure the EOFs below threshold value should be removed. In that case, $E_{l}(n)$ to $E_{5}(n)$ are to be considered for final reconstruction of denoised OFDM signal discarding the first EOF. In fact, in SSA, the first component corresponds to first singular value and represents the important component of the signal. Hence reconsrtuction without $E_{l}(n)$ will not give approprite denoised signal. In such situations at low SNRs, the kurtosis will become a useful measure.

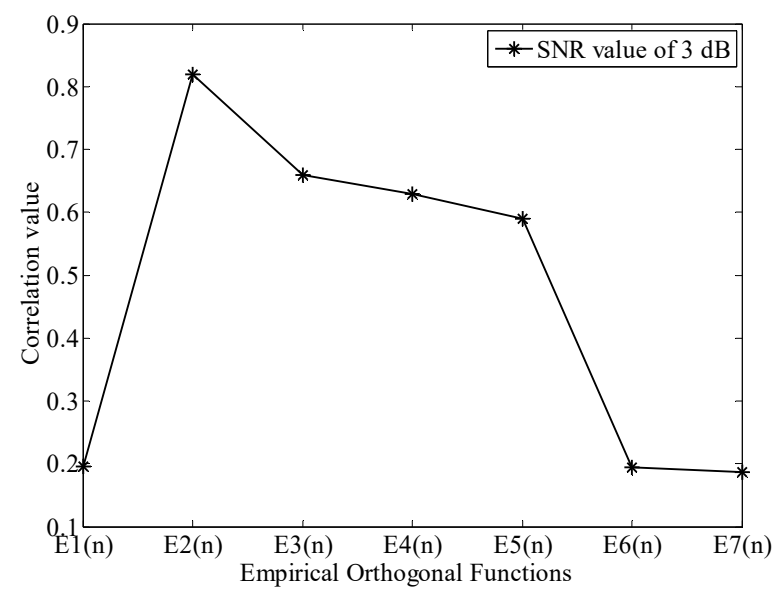

Fig. 5. Correlation values of different EOF with received OFDM symbol for SNR of $3 \mathrm{~dB}$

The mean of kurtosis values is 5.2055, standard deviation is 2.0612 and computed threshold $\sigma_{t h_{-} k}=7.2668$. Hence, the EOFs having kurtosis values below $\sigma_{t_{-} k}=7.2668$ will be considered. This selection will include the first EOF. Finally, the denoised signal is reconstructed using $E_{l}(n)$ to $E_{5}(n)$. So kurtosis is a useful mesure for selectionof EOFs at low values of SNR.

To establish this concept, further simulations are carried out at different SNRs. The result presented in Fig. 6, reveals that correlation is capable of identifying useful EOFs only for $\mathrm{SNR} \geq 12 \mathrm{~dB}$, whereas for SNRs $<12 \mathrm{~dB}$, kurtosis will be useful measure to identify useful EOFs.

The resulting denoised signal $y_{d}(n)$ will used for further precessing for channel estimation by proposed method. One of the prime merits of the proposed SSA based technique is that use of overlapping data segments.

The constellation methods such as BPSK, QPSK, 16-QAM and 64-QAM are considered for BER computation. The propsed SSA based technique performance is compared with LSE [3], MMSE [7], SVD [1] methods. The simulations are presented in Fig. 7 to Fig. 8 to show the superiority of the proposed method. It can be seen from Fig. 7, under BPSK 
constellation that there is a linear trend among methods for all SNR vaues. For a BER of $10^{-3}$, the proposed SSA based signal processing method provides an improvement of $7 \mathrm{~dB}$ compared to LS, $4 \mathrm{~dB}$ compared to MMSE and $1.5 \mathrm{~dB}$ for BPSK constellation.

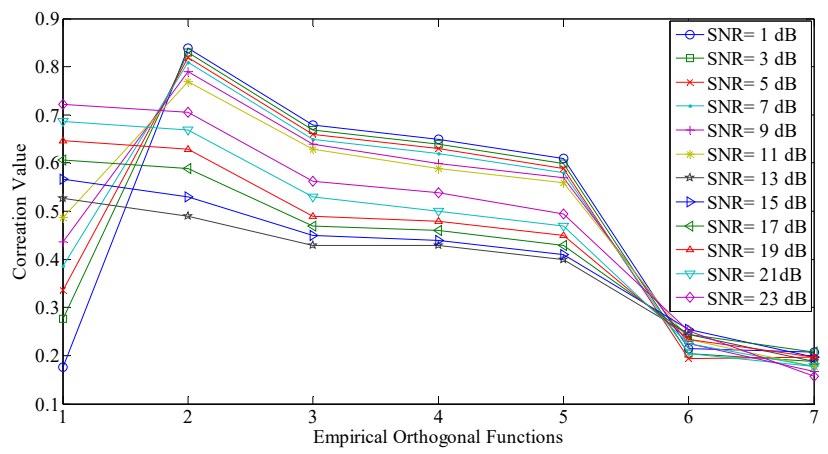

Fig. 6. Correlation values of EOFs with received OFDM symbol for different values SNR

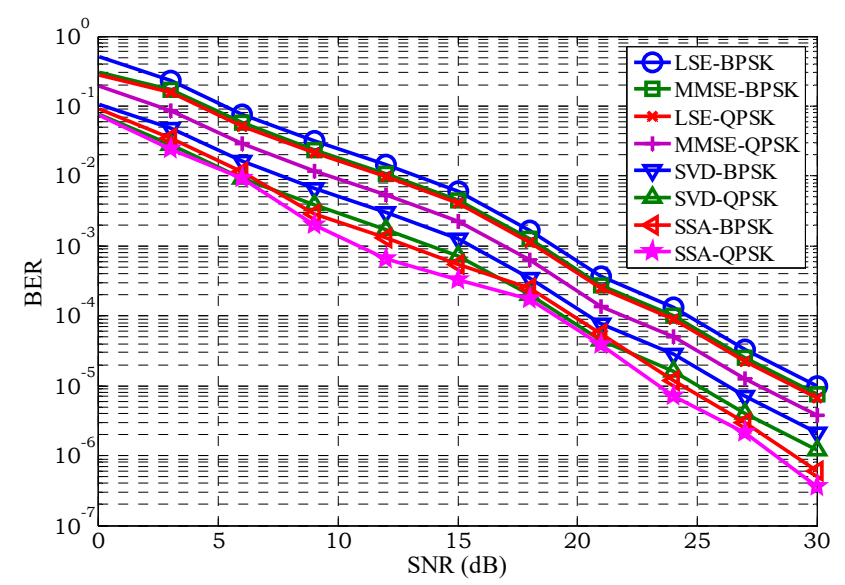

Fig. 7. BER plot of OFDM transceiver using BPSK and QPSK modulation Schemes

In Fig. 7, for QPSK constellation, for small SNR values there is a extra enhancement than higher SNR values. Here for a BER of $10^{-3}$, the proposed method outperforms the LSE method by $8 \mathrm{~dB}$, MMSE by $6 \mathrm{~dB}$ and SVD by $3 \mathrm{~dB}$. For 16QAM, the proposed method has shown an improved performance by $6 \mathrm{~dB}$ over LSE, $5 \mathrm{~dB}$ over MMSE and $3 \mathrm{~dB}$ over SVD based methods as depicted in Fig. 8.

From Fig. 8 for 64 QAM constellation, it can be seen an enhancement of $7 \mathrm{~dB}, 4 \mathrm{~dB}$ and $2 \mathrm{~dB}$ respectively as compared to LSE, MMSE and SVD based methods. All the simulations aimed at studying the efficacy of the proposed method have clearly demonstrated that comparing to LSE, MMSE and SVD based methods, the propsed SSA based method provides better performance.

In addition, the mean square error (MSE) of CIR estimation is also evalauated and shown in Fig. 9, indicates an improvement for SSA based technique.

With the addition of de-noising stage the performance of presented SSA based technique is further improved. The results presented in Fig. 10 establish the fact that it effectively reduces the signal noise, and hence improves the estimation performance. With this denoising approach, the MSE of channel estimator has been further improved which translated to approximately $1 \mathrm{~dB}$ improvement in SNR at the receiver.

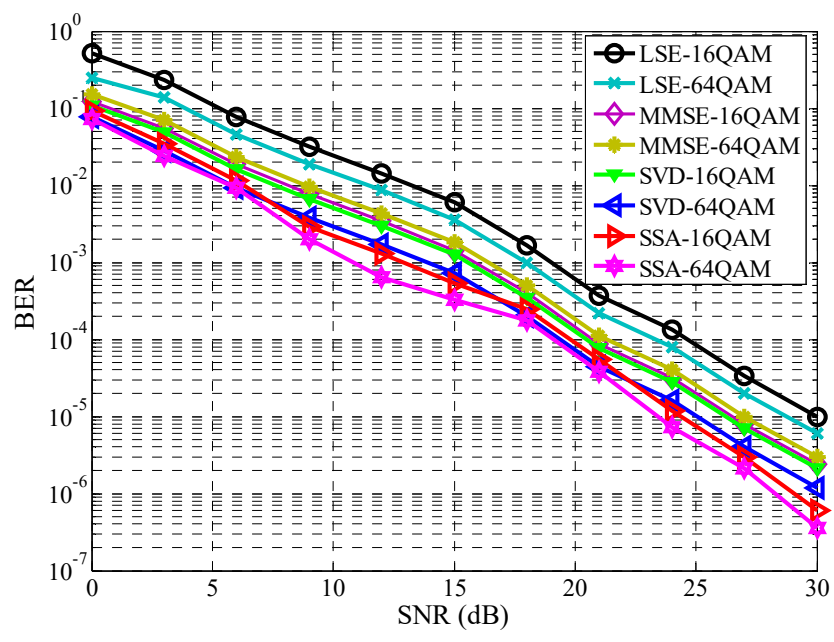

Fig. 8. Comparison of BER: 16 QAM and 64-QAM constellation

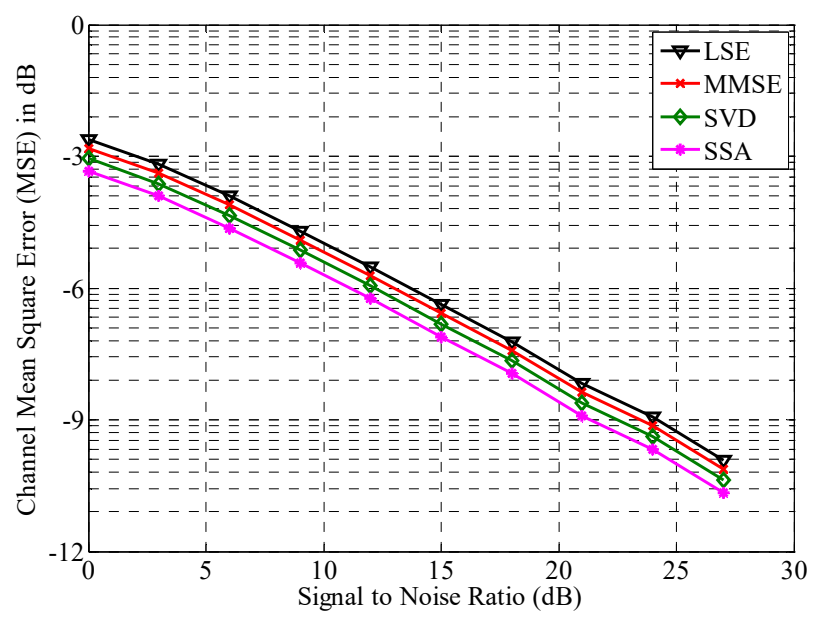

Fig. 9. Channel MSE comparison

In addition to outperforming the existing methods, this additional $1 \mathrm{~dB}$ SNR improvement is achieved at the cost of computations involved during the de-noising process.

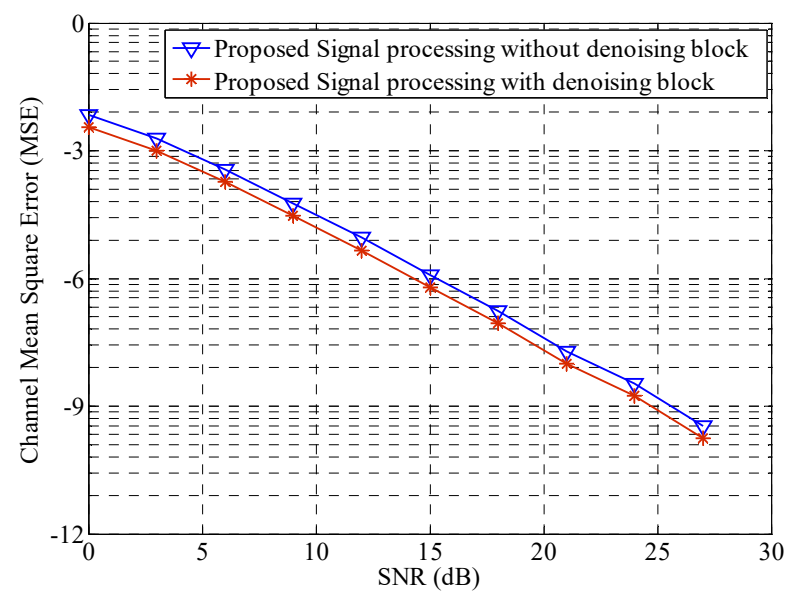

Fig. 10. Channel MSE evaluation with and without inclusion of de-noising block 
The normalized root mean square error (NRMSE) of channel impulse response is computed for different channel environments, to further launch the effectiveness of the presented SSA based technique.

$N R M S E(d B)=20 \log _{10}\left[\sqrt{\frac{\sum_{n=0}^{N}\left[h_{C I R}(n)-\hat{h}_{C I R}(n)\right]^{2}}{\sum_{n=0}^{N}\left[h_{C I R}(n)\right]^{2}}}\right]$

where $N$ is length of the CIR, $h_{C I R}(n)$ and $\hat{h}_{C I R}(n)$ is assumed and estimated impulse response respectively.

TABLE IV. NRMSE (DB) COMPUTED UNDER DIFFERENT CHANNEL ENVIRONMENTS

\begin{tabular}{llllll}
\hline \hline & I & II & III & IV & V \\
\hline \hline NRMSE (dB) & -8.29 & -5.63 & -6.23 & -8.41 & -6.25 \\
\hline \hline
\end{tabular}

The evaluated NRMSE values, as shown in Table IV, are very low and indicate the effectiveness of the proposed SSA based technique for OFDM CE.

\section{CONCLUSION}

A method based on singular spectrum analysis (SSA) has been presented here for OFDM channel estimation (CE). With the use of overlapping data segments in the processing steps of SSA makes it useful for OFDM CE under fast fading conditions. In the proposed algorithm, the initial $\mathrm{CE}$ is computed with the aid of traditional LMMSE algorithm, and then further channel is evaluated by considering the low rank eigenvalue approximation of channel matrix using SSA. The BER and channel error MSE results were compared with wellestablished CE methods such as LSE, MMSE and SVD. Simulation results revealed that the method attained $7 \mathrm{~dB}$ improvement compared to common LSE, $5 \mathrm{~dB}$ over MMSE and almost $3 \mathrm{~dB}$ improvement over SVD. SSA used here for two purposes i.e. $\mathrm{CE}$ and de-noising, which further improved estimation accuracy. As part of de-noising purpose, SSA decomposed the received OFDM symbol into different empirical orthogonal functions (EOFs) as in the order of singular values and noisy reduced signal was combined with either highly correlated EOFs or kurtosis computed EOFs. Correlated EOFs were identified based on either with threshold on correlation or with threshold on kurtosis calculated between received signal and EOFs. It was demonstrated that the correlation coefficients worked well in identifying useful EOFs only up to moderate SNR $\geq 12 \mathrm{~dB}$. For low $\mathrm{SNR}<12 \mathrm{~dB}$, kurtosis was found to be a useful measure for identifying the useful EOFs. With this de-noising approach, the MSE of channel estimator has been further improved which translated to approximately $1 \mathrm{~dB}$ improvement in signal-to-noise ratio at the receiver. In addition to outperforming the existing methods, this additional $1 \mathrm{~dB}$ SNR improvement was achieved at the cost of computations involved during the de-noising process. Complete simulation analysis under all fading channels revealed the efficacy of the proposed system. The real time computational complexity analysis will fully explore effectiveness of the proposed method will be the possible future scope.

\section{REFERENCES}

[1]O. Edfors, M. Sandell, J. J. van de Beek, S. K. Wilson, P.O. Borjesson: OFDM channel estimation by singular value decomposition, IEEE Trans. on Communications, Vol. 46, No. 7, pp. 931-939, July 1997. (DOI: 10.1109/26.701321)

[2]H. Liu, G. Li, "OFDM Fundamentals" in OFDM based broadband wireless networks: Design and Optimization, John Wiley \& Sons 2006.

[3]X. N. Fernando, "Fi-Wi for 4G, 5G, and OFDM Wireless Networks," in Radio over Fiber for Wireless Communications: From fundamentals to advanced topics, Wiley IEEE: John Wiley \& Sons 2014.

[4]X. Dong, W. S. Lu, A. C. K. Soong: Linear interpolation in pilot symbol assisted channel estimation for OFDM, IEEE Trans. on Wireless Communications, Vol. 6, No.5, pp. 1910-1920, May 2007. (DOI: 10.1109/TWC.2007.360392)

[5]Y. S. Lee, H. C. Shin, H. N. Kim: Channel estimation based on timedomain threshold for OFDM systems, IEEE Trans. on Broadcasting. Vol. 55, No.3, pp.656-662, Sep. 2009. (DOI: 10.1109/TBC.2009. 2027575)

[6]L. Dai, Z. Wang, Z. Yang: Spectrally efficient time-frequency training OFDM for mobile large-scale MIMO systems, IEEE Journal of Selected Areas in Communications, Vol. 31, No.2, pp. 251-263, Feb. 2013. (DOI: 10.1109/JSAC.2013.130213)

[7]W. Zhang, X. G. Xia, P. C. Ching: Optimal training and pilot pattern design for OFDM systems in Rayleigh fading, IEEE Trans. on Broadcasting, Vol. 52, No. 4, pp. 505-514, Dec. 2006. (DOI: 10.1109/TBC.2006.884001)

[8]P. S. Rossi, R. R. Muller, O.Edfors: Linear MMSE estimation of timefrequency variant channels for MIMO-OFDM systems, Signal Processing, Vol. 91, No. 5, pp. 1157-1167, May 2011. (DOI: 10.1016/j.sigpro.2010.10.017)

[9]S. Pallaviram, B. Chandra Mohan: A pilot aided channel estimator using DFT based time interpolator for massive MIMO-OFDM systems, International Journal of Electronics and Communications (AEÜ) Vol. 69, pp. 321-327, Jan. 2015. (DOI: 10.1016/j.aeue.2014.10.001)

[10] J. Oliver, R. Aravind, K. M. M. Prabhu: A Krylov subspace based lowrank channel estimation in OFDM systems, Signal Processing, Vol. 90, No. 6, pp. 1861-1872, June 2010. (DOI: 10.1016/j.sigpro.2009.12.004

[11] M. K. Ozdemir, H. Arslan: Channel estimation for wireless OFDM systems, IEEE Communication Surveys \& Tutorials, Vol. 9, No.7, pp. 18-48, 2007. (DOI: 10.1109/COMST.2007.382406)

[12] Liu. Y., Tan, Z., Hu, H., Cimini, L.J., Ye Li, G: Channel estimation for OFDM, IEEE Communications Surveys \& Tutorials, Vol. 16, No. 4, pp. 1891-1908, 2014. (DOI: 10.1109/COMST.2014.2320074)

[13] B. Mokhtar, D. A. Bouzidi: New design of pilot patterns for joint semiblind estimation of CFO and channel for OFDM systems, International Journal of Electronics \& Communications (AEÜ), Vol. 69, No. 4, pp. 759-764, June 2015. (DOI: 10.1016/J.AEUE.2015.01.008)

[14] M. N. Seyman, N. Taspinar: Channel estimation based on neural network in space time block coded MIMO-OFDM system, Digital Signal Processing, Vol. 23, No. 1, pp. 275-280, Jan. 2013. (DOI: 10.1109/IBC AST47879.2020.9044539)

[15] W. Zhang, F. Gao, Q. Yin: Blind channel estimation for MIMO-OFDM systems with low order signal constellation, IEEE Communication Letters, Vol. 19, No.3, pp. 499-502, Mar. 2015. (DOI: 10.1109/LCOM M.2015.2393889

[16] S. Beygi, U. Mitra: Multi-scale multi-lag channel estimation using low rank approximation for OFDM, IEEE Trans. on Signal Processing, Vol. 63, No.18, pp. 4744-4755, June 2015. (DOI: 10.1109/TSP.2015. 2449266)

[17] Ann-Chen Chang: Using ICA to improve blind subspace-based channel estimation for OFDM system under unknown noise fields, International Journal of Electronics and Communication (AEÜ), Vol. 69, No. 1, pp. 449-454, Jan. 2015. (DOI: 10.1016/j.aeue.2014.08.013

[18] V. Savaux, Y. Louët, M. Djoko-Kouam, A. Skrzypczak: Artificial channel aided LMMSE estimation for time-frequency selective channels in OFDM context, Signal Processing, Vol. 93, No. 9, pp. 2369-2380, Sep. 2013. (DOI: 10.1016/j.sigpro.2013.03.006) 
[19] S. Pallaviram, B. Chandra Mohan: An eigen-select denoising threshold for channel estimation in OFDM systems, International Journal of Communication Systems, Vol. 30, No.3, pp. 2971, Feb. 2017. (DOI: 10.1002/dac.2971)

[20] S. Pallaviram, B. Chandra Mohan: A survey on OFDM channel estimation techniques based on denoising strategies, Engineering Science and Technology, an International Journal, Vol. 20, No.2, pp. 629-636, Apr. 2017. (DOI: 10.1016/j.jestch.2016.09.011)

[21] V. Savaux, Y. Louët: LMMSE Channel Estimation in OFDM Context, A Review, IET Signal Processing, Vol. 11, No. 2, pp. 123 - 134, April 2017. (DOI: 10.1049/iet-spr.2016.0185)

[22] A. Munshi and S. Unnikrishnan, "Performance analysis of compressive sensing based LS and MMSE channel estimation algorithm," in Journal of Communications Software and Systems, vol. 17, no. 1, pp. 13-19, Feb. 2021, (DOI: 10.24138/jcomss.v17i1.1084)

[23] T. Yang, J. Shao, Y. Huang and R. Malekian, "A method of multicomponent signal detection based on differential nonlinear mode decomposition," in Journal of Communications Software and Systems, vol. 14, no. 2, pp. 171-179, July 2018. (DOI: 10.24138/jcomss. v14i2.514)

[24] E. H. Krishna, K. Sivani, K. A. Reddy: New channel estimation method using singular spectrum analysis for OFDM systems, Wireless Pers. Communications, Vol. 101, pp. 2193-2207, Aug. 2018. (DOI: 10.1007/s11277-018-5811-5)

[25] D. S. Broomhead, G. King: Extracting qualitative dynamics from experimental data, Physica D, Vol. 20, 217-236, 1986. (DOI: 10.1016/0167-2789(86)90031-X)

[26] Allen M., Smith L.A., Optimal filtering in singular spectrum analysis, Phys. Lett., vol. 234, pp. 419-428, 1997. (DOI: 10.1016/S03759601(97)00559-8)

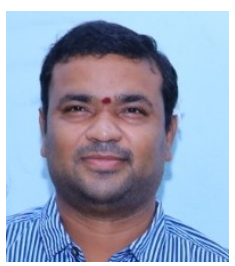

E. Hari Krishna was born in Warangal, India, in 1983. He received B. Tech degree from Jawaharlal Nehru Technological University (JNTU), Hyderabad in 2004 and M. Tech degree from Kakatiya University (KU), Warangal, in 2009 and $\mathrm{PhD}$ from JNTUH, Hyderabad in 2021. He is currently an Assistant Professor in Department of Electronics \& Communication Engineering, University College of Engineering, KU, Kothagudem, India. His research interests include signal processing for wireless communications, channel estimation in $4 \mathrm{G}$ and $5 \mathrm{G}$ communication system, biomedical signal processing and signal decomposition methods.

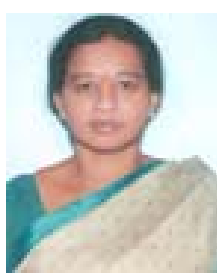

K. Sivani was born in the year 1966. She received her B. Tech degree from Kakatiya University, Warangal, India, M. Tech degree from National Institute of Technology, Warangal, India and her Doctorate from Jawaharlal Nehru Technological University, Hyderabad, India. At present she is working as professor in Electronics and Instrumentation Engineering at Kakatiya Institute of Technology \& Science, Warangal, India.. Her areas of interest include VLSI Design and Biomedical engineering.

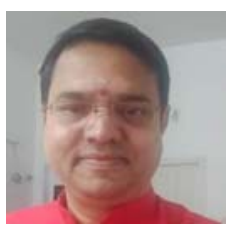

K. Ashoka Reddy was born in Warangal, India, in 1970. He received B. Tech degree from Kakatiya University, Warangal in 1992 and M. Tech degree of Jawaharlal Nehru Technological University (JNTU), Kakinada, India, in 1994, and Ph.D degree in Electrical Engineering from Indian Institute of Technology Madras, India, in 2008. He is currently a Professor with the Department of Electronics and Communication Engineering, Kakatiya Institute of Technology \& Science, Warangal, India. His research interests include instrumentation, biomedical signal processing, signal processing for communication and artificial neural networks. 